\title{
Thermal simulation for continuous-flow PCR system
}

\author{
Shenglan Zeng \\ School of Southeast University \\ of Bio-medical Engineering \\ Nanjing, China \\ 220143725@seu.edu.cn \\ Lingfeng Cai \\ School of Southeast University \\ of Bio-medical Engineering \\ Nanjing, China \\ 220151643@seu.edu.cn
}

\author{
Bingyu Chen \\ School of Southeast University \\ of Bio-medical Engineering \\ Nanjing, China \\ 220153842@seu.edu.cn \\ Quanjun Liu \\ School of Southeast University \\ of Bio-medical Engineering \\ Nanjing, China \\ lqj@seu.edu.cn
}

\begin{abstract}
A continuous-flow PCR system is presented, which based on the microfluidic platform briefly consists of a PMMA chip with a PCR liquid channel, three heating units. Each of them contained a heat conducting block on a commercially available thin PI substrate. In order to ensure temperature uniformity across the PCR temperature zones and minimal cross-talk between the zones, the design of chip and heating units was assisted by COMSOL. The simulation results presented the most suitable thickness for the chip and heat conducting block as well as the height limit for the liquid channel. Within the optimized parameters, our system not only has the advantages of fast amplification rates, but also has the benefits of small thermal mass and low power consumption.
\end{abstract}

\section{Keywords_PCR; microfluidic; COMSOL; Simulation}

\section{INTRODUCTION}

As a sensitive and powerful tool for nucleic acid detection, PCR has been widely used in many fields from basic research to clinical diagnostics for bacterial identification[1], sample preparation for DNA sequencing [2],etc. "Where PCR is really going," says Olivier Harismendy at the University of California, San Diego, "is parallelization and miniaturization." Indeed, researchers are making use of a wide variety of materials and applications toward these goals[3]. Fortunately, MEMS technology are being developed in the past few years, which will represent a central technology in many miniaturized systems used for biological, chemical, and medical applications [4-8].

Many researchers have described various kinds of miniaturized systems for PCR, including plastic chip integrated micro-chambers for multiplex PCR[1], highthroughput droplet digital PCR system for absolute quantitation of DNA Copy number[9], single copy DNA amplification and cell analysis in engineered nanoliter droplets[2, 10], etc. By using these microfluidics devices, the flow speed and precision can be operated because of the small volumes. Other advantages include portability plus the ability to work with smaller samples and fewer reagents. However the speed of the reaction is limited by the inter- temperature transition time. The consumption time of the traditional heating method rely on the speed of heating and cooling of the substrate. Continuous-flow thermal gradient PCR is a new DNA amplification technique that is characterized by periodic temperature ramping with no cyclic hold times. By excluding the substrate from the thermal cycling, lower energy consumption and faster cycling can be achieved[11, 12]. In continuous-flow PCR, the reaction mixture passes through zones of alternating temperature corresponding to denaturation, annealing, and extension[13,14], each cycle could be accelerated[15, 16].

In this work, an all-plastic continuous-flow PCR device is designed and integrated with heating elements. In order to reach higher amplification efficiency, the structure of microchip plus the temperature distribution of heating system were calculated with simulation software COMSOL. Finally, the most suitable parameters of the system were presented to guide the design of chip and heating elements.

\section{MODEL DEFINITION}

\section{A. The Structure of PCR System}

The system based on the microfluidic platform briefly consists of a chip with a PCR liquid channel, three heating units corresponding to denaturation, annealing, and extension zone (Fig.1.A). In order to isolate three constant temperature zones, the chip was made of PMMA for its low heat conductivity. The properties of the PMMA such as easy to optical detection, disposable with low cost and good biocompatibility started a new scope of operation for PCR. The micro channel was engraved by $\mathrm{CNC}$ (Computer Numerical Control) engraving and milling machine, the structure of which defined the cycle number of PCR. The etched PMMA substrate was covered with another undressed PMMA plates with same size, after alignment and fixation, the two plates were bonding on a constant temperature drying boxes. 

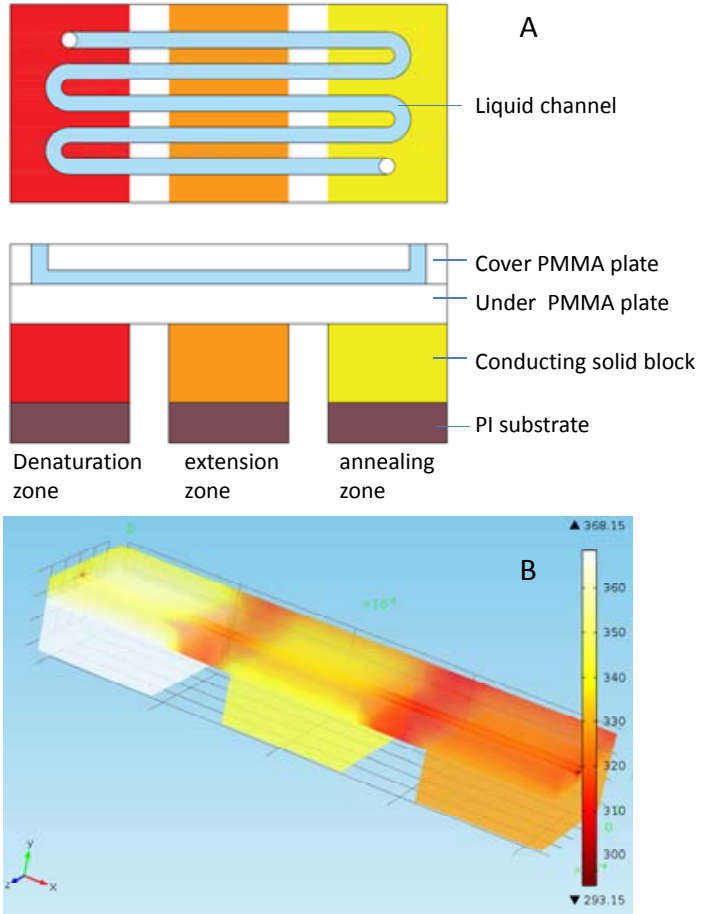

Fig. 1. (A) Schematic of PCR system. (B) The thermal model of the PCR system established by COMSOL.

In this system, a channel passes through three zones corresponding to denaturation, annealing, and extension circularly, a cycle begins with the denaturation zones and passing through the extension zones and annealing zones, at last ended at the extension zones, after that another cycle is started. The heating unit contained a conducting solid metal on a commercially available, very thin $(100 \mu \mathrm{m})$ PI substrate.

In spite of the undisputed advantages of continuous-flow PCR devices, the best structure of chip has not yet been demonstrated. Numerical calculations are performed to demonstrate the most suitable operating parameters such as thickness of channel and chip.

\section{B. Thermal Model}

The model (Fig.1.B) was established using COMSOL. The temperature of PI substrate is controlled by automatic thermal control system, maintaining its temperature stability, thus the PI substrate can simplified as a surface with constant temperature. The liquid channel is simplified into two symmetrical fluids, one is flowing from high temperature to low temperature, another is flowing from low temperature to high temperature, the velocity of them are 8 $\mu \mathrm{l} / \mathrm{min}$. Conjugate heat transfer analysis is employed in the whole geometry, solid analysis is used between chip and heat conduction block while conjugate analysis are apply for chip and fluids. The initial temperature is set at $293.15 \mathrm{~K}$. Boundary surfaces are simultaneously exposed to convective cooling. At last, the model is computed to get a stationary result.

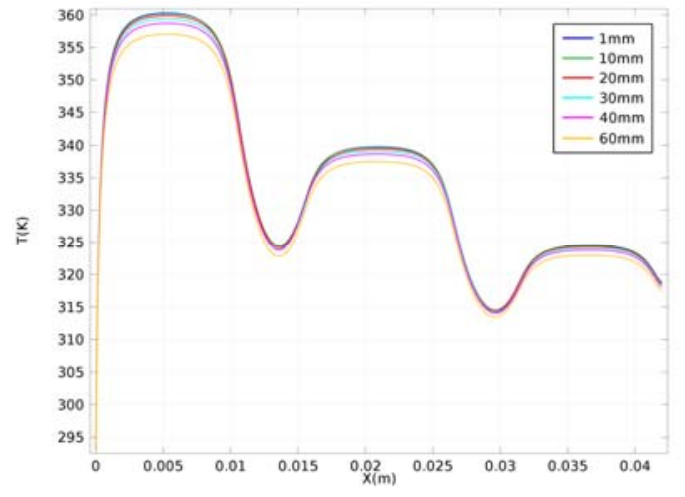

Fig. 2 .Temperature distributions of liquid channel with different thickness of conduting block.

\section{THERMAL ANALYSIS}

\section{A. The Thickness and Material Analysis of Conducting solid block}

The thickness of conducting solid block affect the efficiency of thermal conductivity, the thinner the blocks are, the lower power consumption of the system. However, the chip was supported by conducting block. Numerical calculations were used to find a suitable thickness to meet the demands of high efficiency and high strength. In order to determine the effect of that single factor, we tentatively set copper as the material of the conducting block. The thickness values were scanned from $1 \mathrm{~mm}$ to $60 \mathrm{~mm}$. The results are showed on fig2.

As shown (Fig.2), there is a coincidence on the temperatures of fluid when thickness is $1 \mathrm{~mm}$ and $10 \mathrm{~mm}$. When thickness is superior to $10 \mathrm{~mm}$, the temperature of fluid decreased within the increase of thickness. Therefore the thickness of conducting solid block should be selected below $10 \mathrm{~mm}$. We choose the thickness of $4 \mathrm{~mm}$ for the follow-up experiments.

Another factor influences the efficiency of heat conduction is the material. Four kinds of materials which are regularly employed in PCR conduction system were calculated in this study. The features of the materials are shown in the following table (tab1).

It can be seen from the Fig.3, when using aluminum, copper and silver as the material of conducting block, the temperature of the fluid channel are basically identical, while the usage of glass showed significantly lower temperature. To conclude, metal is perfect suit for the heat conduction block. In order to reduce the cost, we can choose the cheapest aluminum as the material of heat conducting block, its performance is basically the same as the silver when the thickness is $4 \mathrm{~mm}$.

\begin{tabular}{|l|l|l|l|}
\hline \multicolumn{2}{|c}{ TABLE I. FEATURES OF MATERIALS } \\
\hline Materials & $\begin{array}{l}\text { Density/ } \\
(\mathbf{k g} / \mathbf{m} \wedge \mathbf{3})\end{array}$ & $\begin{array}{l}\text { Thermal conductivity } \\
/(\mathbf{W} /(\mathbf{m} * \mathbf{K}))\end{array}$ & $\mathbf{C / ( J / ( \mathbf { k g } ^ { * } \mathbf { K } ) )}$ \\
\hline $\mathrm{Cu}$ & 8960 & 401 & 384 \\
\hline $\mathrm{Al}$ & 2700 & 237 & 904 \\
\hline $\mathrm{Ag}$ & 1050 & 429 & 235 \\
\hline Glass & 2200 & 1.1 & 480 \\
\hline
\end{tabular}




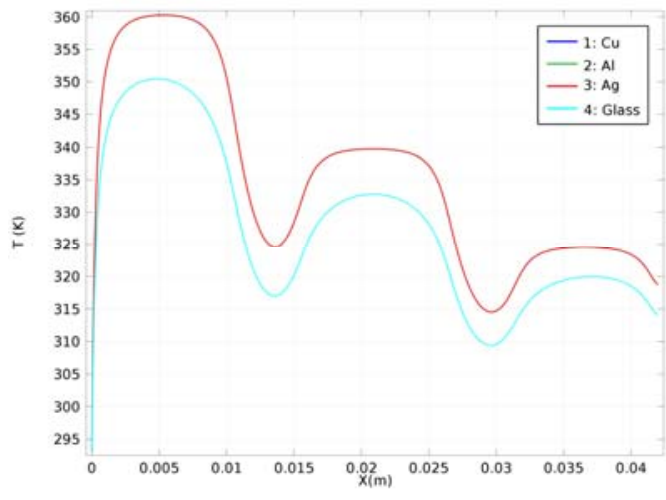

Fig. 3. Temperature distribution of channel with different conducting materials.

\section{B. The Most appropriate thickness for the Cover PMMA plate}

It's known that the temperature distribution of channel will be more uniform if the distance between the channel and conducting block is shorter. Thus the thinner PMMA plate adjoins the conducting block is better. We tentatively use the PMMA plate thick of $1 \mathrm{~mm}$, which is the minimum thickness commercially available. The only parameter need to be optimized is the thickness of the cover plate.

Thicknesses from $0.6 \mathrm{~mm}$ to $1.4 \mathrm{~mm}$ were selected for scanning while the temperature of environment varied from $273.15 \mathrm{k}$ to $303.15 \mathrm{k}$. In order to visually observe the trends, the temperature of channel on denaturation zones minus their average temperature is on the vertical axis. It is clearly showed that the trends of temperature along with the thickness of cover plate are similar in different environmental temperature (Fig.5). Temperatures peak when the thickness of cover plate is $1 \mathrm{~mm}$, which means it have the function of heat insulation. It will hold the temperature from thermal radiation and not too thick to cause excessive heat losses. When the thickness is greater than $1 \mathrm{~mm}$, the temperature will significantly decrease with the increase of thickness. So $1 \mathrm{~mm}$ is the most appropriate thickness for the cover PMMA plate.

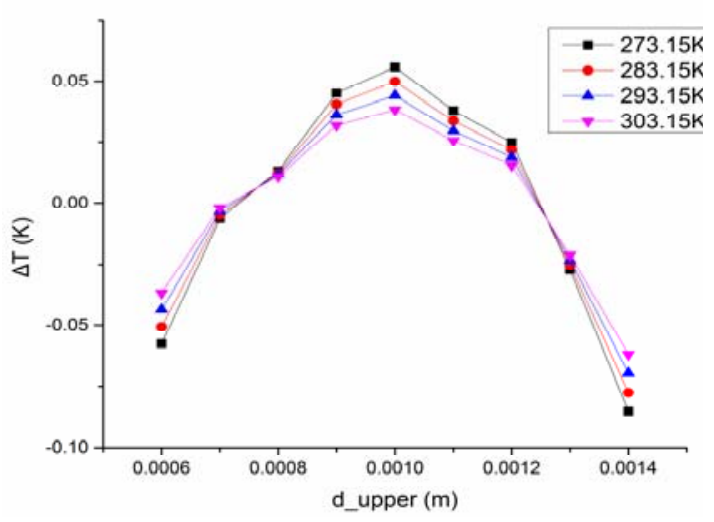

Fig. 4. Trends of temperature along with the thickness of cover plate.

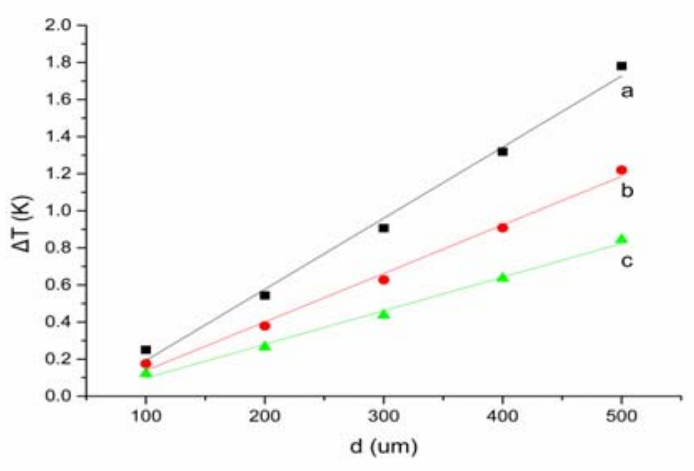

Fig. 5.Temperature gradient variation with channel height. (a) denaturation zone.(b) extension zone (C)annealing zone.

\section{The Most appropriate height for the liquid channel}

The width of liquid channel is determined by the machining precision, the width should be higher than the minimum machining precision. We set the width of the liquid channel at $200 \mu \mathrm{m}$ which is regularly selected in micro channels. As for the height of liquid channel, the minimum value is limited by the machining precision while the maximum value is limited by the temperature uniformity. In order to guarantee the uniformity of amplification, the temperature gradient of the liquid in the channel should be less than $1.0^{\circ} \mathrm{C}$ at each temperature zone. Five height values were calculated to find the relationship between the temperature gradient and the height.

As shown (Fig.4), a linear correlation was found between the temperature difference and the height of fluid channel. When the height of fluid channel is small, the temperature difference between the top and bottom surface of the channel is small, achieving better temperature uniformity. In order to keep the temperature gradient under $1.0{ }^{\circ} \mathrm{C}$ at each temperature zone, the height of the liquid channel should be smaller than $300 \mu \mathrm{m}$.

\section{Simulation for minimal Thermal cross-talk}

In this system, three zones corresponding to denaturation, annealing, and extension played an important role in polymerase chain reaction, the temperature of which need to be accurate controlled. Three heaters were put side by side under the chip with particular interval. If the gap between heaters is enough long, the three zones are isolated without mutual influence. In order to reduce the volume of the chip and lower the power consumption, the gap between heaters should be optimized. The separation gap between the heating zones was determined through simulation for minimal thermal cross-talk.

The y-axis of Fig.6.A represents the absolute value of inlet temperature minus middle temperature of extension zones. The lower value of $y$-axis means the temperature of extension zones will be faster to reach the plateau and the effective time for extension will be increased. However the minimal thermal cross-talk is affected by convection coefficient called $\mathrm{h}$ and the ambient temperature. If $\mathrm{h}$ is low, the minimal thermal cross-talk will increase. The minimal 


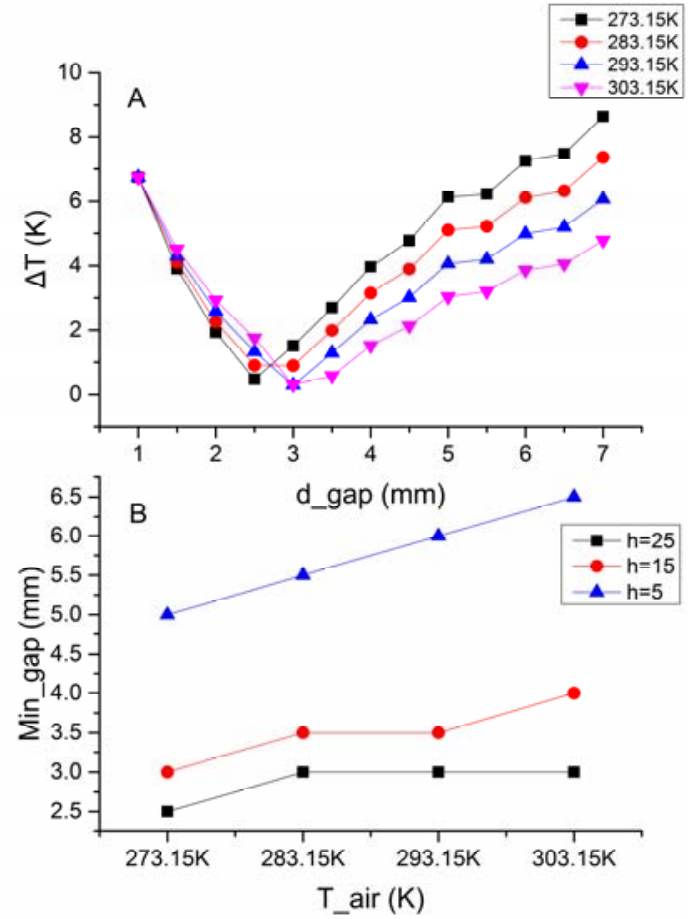

Fig. 6. (A) Temperature differences of extension zones along with intervals, when $\mathrm{h}=15$. (B) Minimal thermal cross-talk in different ambient temperature and $\mathrm{h}$.

thermal cross-talk can be determined refer to Fig.6.B. When the gap between each zone is longer than $3.5 \mathrm{~mm}$ at $293.15 \mathrm{k}$ and $\mathrm{h}=15$, there is no significant thermal cross-talk between the zones.

\section{CONCLUSION}

The design and fabrication of a continuous-flow PCR chip is presented. Our system not only has the advantages of fast amplification rates, but also has the benefits of small thermal mass and low power consumption. The chip design was assisted by conjugate heat transfer analysis to ensure temperature uniformity across the PCR temperature zones and minimal cross-talk between the zones. We recommend a range of operational parameters such as the thickness of conducting solid block should be less than $10 \mathrm{~mm}$, the thickness of the cover PMMA plate should be $1 \mathrm{~mm}$, the height of the channel should be less than $300 \mu \mathrm{m}$. It is perfect for cost saving to use aluminum as the material of conducting solid block. The gap between heaters is affected by the ambient temperature and convection coefficient. Generally $3.5 \mathrm{~mm}$ could be the suitable length of the interval.

It is the first time to report the best structure from chip to heat conducting block for continuous-flow PCR system, these results have profound guiding significance for the design of chip and heaters for continuous-flow PCR system.

\section{Acknowledgment}

The work was supported the National Natural Science Foundation of China $(61372031,61071050)$.

\section{References}

[1] Y. Xu, H. Yan, Y. Zhang, K. Jiang, Y. Lu, Y. Ren, et al., "A fully sealed plastic chip for multiplex PCR and its application in bacteria identification," Lab on a Chip, vol. 15, pp. 2826-2834, 2015.

[2] P. Kumaresan, C. J. Yang, S. A. Cronier, R. G. Blazej, and R. A. Mathies, "High-throughput single copy DNA amplification and cell analysis in engineered nanoliter droplets," Analytical chemistry, vol. 80, pp. 3522-3529, 2008.

[3] M. Baker, "Clever PCR: more genotyping, smaller volumes," nature methods, vol. 7, pp. 351-356, 2010.

[4] C. Zhang, J. Xu, W. Ma, and W. Zheng, "PCR microfluidic devices for DNA amplification," Biotechnology advances, vol. 24, pp. 243-284, 2006

[5] H. Zhang, G. Jenkins, Y. Zou, Z. Zhu, and C. J. Yang, "Massively parallel single-molecule and single-cell emulsion reverse transcription polymerase chain reaction using agarose droplet microfluidics," Analytical chemistry, vol. 84, pp. 3599-3606, 2012.

[6] T. Chen, Y. Jia, C. Dong, J. Gao, P.-I. Mak, and R. P. Martins, "Sub-7-second genotyping of single-nucleotide polymorphism by high-resolution melting curve analysis on a thermal digital microfluidic device," Lab on a Chip, 2016.

[7] X. Bi, G. Adriani, Y. Xu, S. Chakrabortty, G. Pastorin, H. K. Ho, et al., "Gene Detection in Complex Biological Media Using Semiconductor Nanorods within an Integrated Microfluidic Device," Analytical chemistry, vol. 87, pp. 10292-10298, 2015.

[8] P. Liu and R. A. Mathies, "Integrated microfluidic systems for high-performance genetic analysis," Trends in biotechnology, vol. 27, pp. 572-581, 2009.

[9] B. J. Hindson, K. D. Ness, D. A. Masquelier, P. Belgrader, N. J. Heredia, A. J. Makarewicz, et al., "High-throughput droplet digital PCR system for absolute quantitation of DNA copy number," Analytical chemistry, vol. 83, pp. 8604-8610, 2011.

[10] B. Lam, Z. Fang, E. H. Sargent, and S. O. Kelley, "Polymerase chain reaction-free, sample-to-answer bacterial detection in 30 minutes with integrated cell lysis," Analytical chemistry, vol. 84, pp. 21-25, 2011.

[11] N. Crews, C. Wittwer, and B. Gale, "Continuous-flow thermal gradient PCR," Biomedical microdevices, vol. 10, pp. 187-195, 2008.

[12] D. Moschou, N. Vourdas, G. Kokkoris, G. Papadakis, J. Parthenios, S. Chatzandroulis, et al., "All-plastic, low-power, disposable, continuous-flow PCR chip with integrated microheaters for rapid DNA amplification," Sensors and Actuators B: Chemical, vol. 199, pp. 470-478, 2014.

[13] Y. Schaerli, R. C. Wootton, T. Robinson, V. Stein, C. Dunsby, M. A. Neil, et al., "Continuous-flow polymerase chain reaction of single-copy DNA in microfluidic microdroplets," Analytical chemistry, vol. 81, pp. 302-306, 2008.

[14] J. A. Kim, J. Y. Lee, S. Seong, S. H. Cha, S. H. Lee, J. J. Kim, et al., "Fabrication and characterization of a PDMS-glass hybrid continuous-flow PCR chip," Biochemical Engineering Journal, vol. 29, pp. 91-97, 2006.

[15] M. Hashimoto, P.-C. Chen, M. W. Mitchell, D. E. Nikitopoulos, S. A. Soper, and M. C. Murphy, "Rapid PCR in a continuous flow device," Lab on a Chip, vol. 4, pp. 638-645, 2004.

[16] C. Gärtner, R. Klemm, and H. Becker, "Methods and instruments for continuous-flow PCR on a chip," in MOEMS-MEMS 2007 Micro and Nanofabrication, 2007, pp. 646502-646502-8. 